\title{
EL PALAU GÜELL DE BARCELONA. LA CONSTRUCCIÓN DE UNA IDEA ESPACIAL
}

\author{
(THE PALAU GÜELL OF BARCELONA. THE CONSTRUCTION OF A SPATIAL IDEA)
}

Antoni González Moreno-Navarro, Arquitecto.

Jefe del Servicio del Patrimonio Arquitectónico de la Diputación de Barcelona. España

\section{RESUMEN}

A modo de introducción de los artículos dedicados a los aspectos constructivos del Palau Güell, el autor de este artículo - director de los trabajos de restauración - traza las caracteristicas esenciales del edificio, una construcción singular en la que las dependencias se ordenan alrededor de un espacio central de marcada directriz vertical que asume las funciones propias del patio central de tradición mediterránea y crea secuencias espaciales y sensoriales que multiplica las reducidas dimensiones del edificio. Para el autor, parece claro que en esta obra de juventud, la estructura no es para Gaudí más que un medio al servicio de la construcción de una idea espacial.

\section{SUMMARY}

As an introduction to the articles dedicated to the constructive aspects of the Palau Güell, the author of this article - director of the restoration works - traces the essential characteristics of the building, a unique construction where the rooms are arranged around a vertically oriented central space which adopts the functions of a traditional Mediterranean patio and creates spatial and sensorial sequences which multiply the reduced dimensions of the building. For the author, it seems clear that in this work, the structure for Gaudi was a medium at the service of the construction of a spatial idea.
Este edificio singular - concebido inicialmente como lugar de reuniones sociales, conciertos privados y residencia de invitados - fue encargado a Gaudí por el conde Güell como ampliación de la residencia que la familia poseía en la vecina Rambla. Un pasillo o galería cubierta, todavía existente, uniria las plantas nobles de ambos edificios. Como consecuencia del estudio del arquitecto, el promotor hubo de comprar una nueva finca para ampliar el solar previsto.

Antoni Gaudí, que tenía 34 años cuando proyectó esta obra - conviene no olvidarlo al enjuiciarla-, dio en ella muestras de concebir la arquitectura, tanto en los aspectos espaciales como en la decoración, de una manera personal. Rompe con la convención de los órdenes arquitectónicos, se aparta de la arquitectura clasicista imperante en Europa, y si bien se inspira de forma evidente en la concepción espacial y formal de la arquitectura hispano-musulmana - con una actitud que tiene poco que ver no obstante con los historicismos eclécticos que empezaban a imponerse-, introduce ya

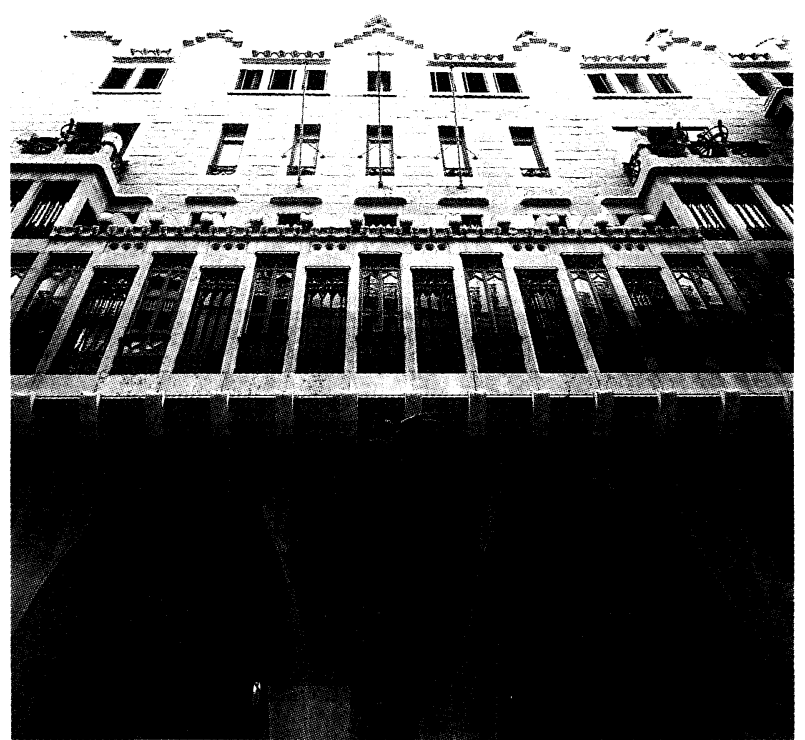

Fig. 1.- Fachada a la calle Nou de la Rambla. (Foto Joan Francés noviembre de 1984)

http://informesdelaconstruccion.revistas.csic.es 


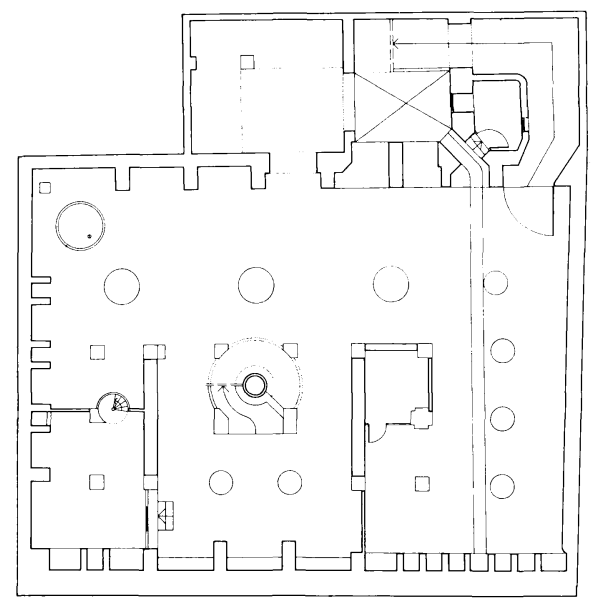

Fig. 2.-Planta subterránea. Cuadras, almacenes y cuarto del cochero. En el centro, la rampa peatonal helicoidal. En la parte superior, la rampa de caballerías.

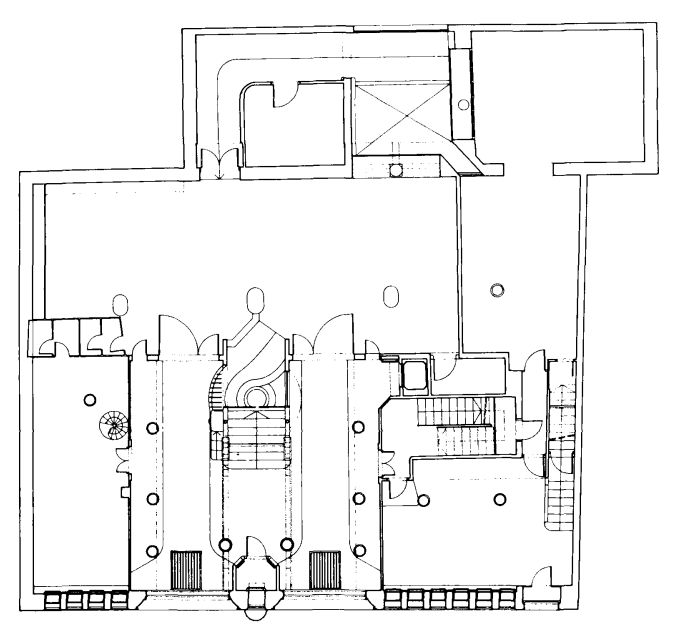

Fig. 3.-Planta baja. Acceso, cocheras, vestibulo, escalera princı pal, porteria y almacenes.

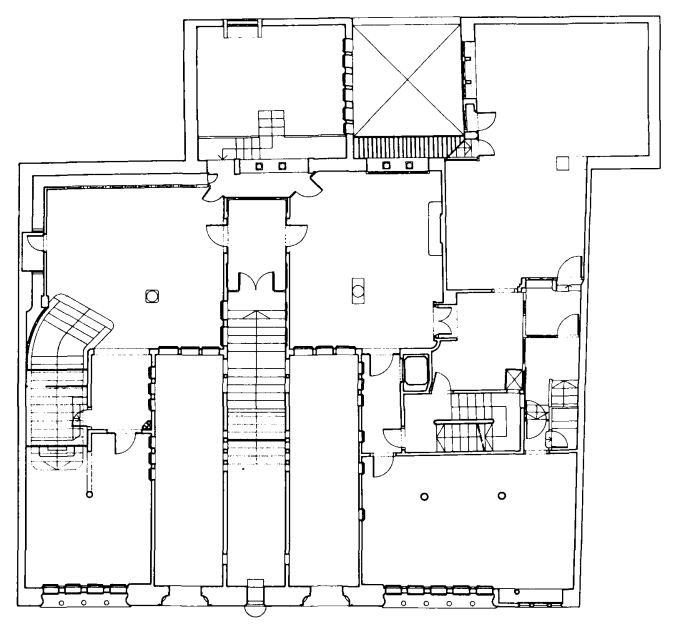

Fig. 4.-Entresuelo. Despacho del señor Güell, biblioteca, sala de descanso, segundo tramo de la escalera principal.

(c) Consejo Superior de Investigaciones Científicas Licencia Creative Commons 3.0 España (by-nc)

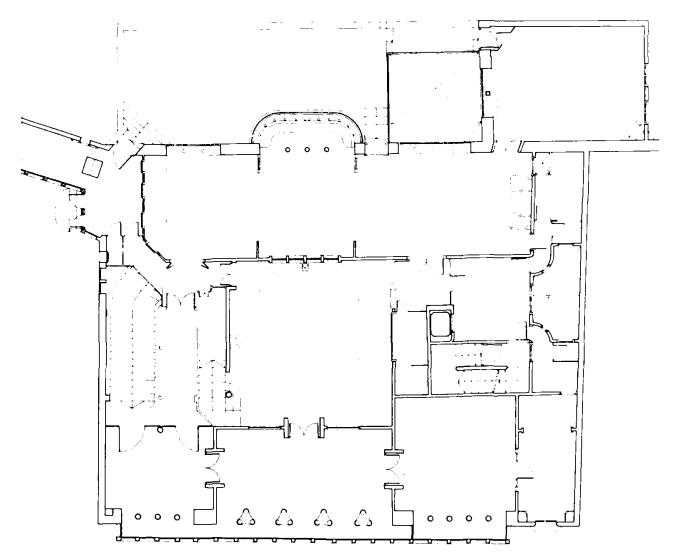

Fig. 5.- Planta noble. Crujia norte: vestíbulo, sala de paso, visitas y tocador. Centro: salón-capilla, órgano. Crujía sur: sala de confianza y comedor. Anexo: sala de billar y taller de dibujo. Medianera de la izquierda: corredor de enlace con la casa Güell de la Rambla.

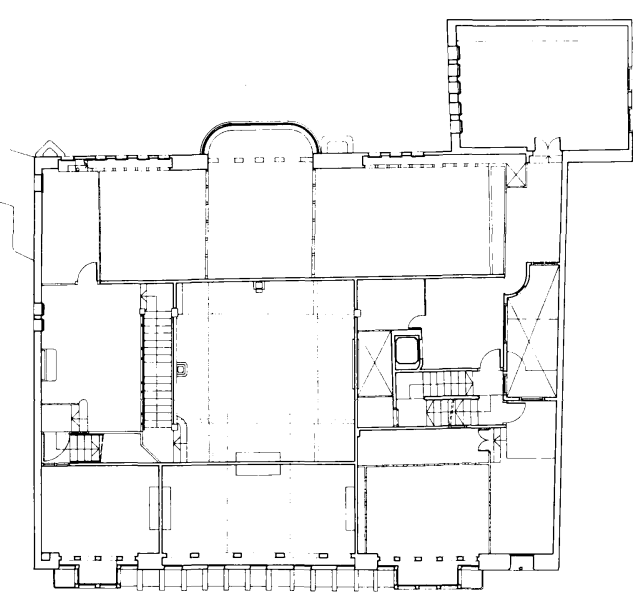

Fig. 6.-Entreplanta. Sala y miranda del salón.

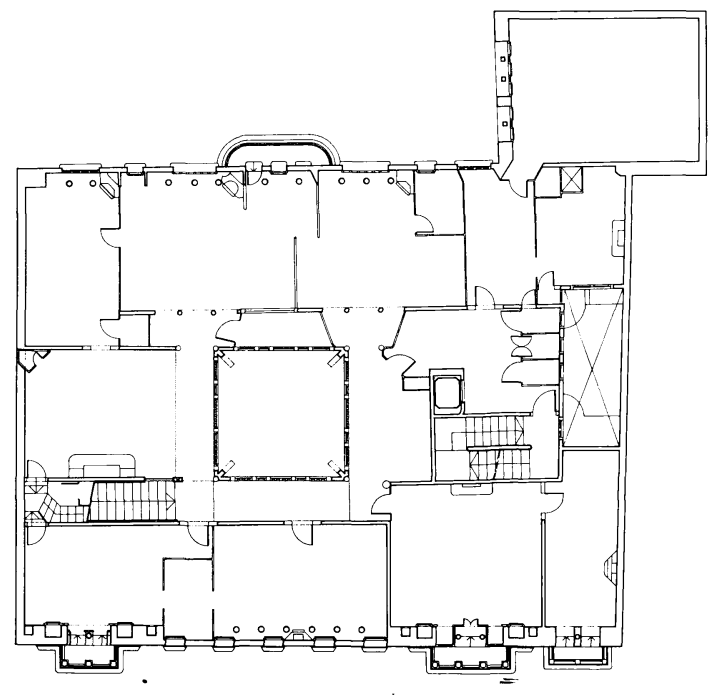

Fig. 7.-Planta segundo piso. Crujia norte: demitorio principal, sala de estudio, dormitorios. Centro: sala de confianza, aseos, tocador. Crujía sur: dormitorios y tocadores.

http://informesdelaconstruccion.revistas.csic.es 
un buen número de aportaciones propias que constituyen la base del repertorio que hará de Gaudí uno de los arquitectos formalmente más renovadores de la arquitectura moderna.

Las dependencias de este peculiar palacio urbano - edificado en un barrio que ya sufría una importante degradación social - se ordenan alrededor de un espacio central, dando en cierta manera la espalda a la calle (la gran tribuna más que un mirador es una ampliación del espacio interior) y al patio de manzana, más degradado si cabe que la calle (las celosías de la fachada posterior se antojan como protecciones de vistas no deseadas más que como protecciones del sol). Ese gran espacio central de marcada directriz vertical (ochenta metros cuadrados de planta por casi veinte de altura) asume - con singular protagonismo compositivo - las funciones propias del patio central de tradición mediterránea. Por otra parte, las secuencias espaciales y sensoriales que crea, multiplica varias veces las reducidas dimensiones del edificio que ocupa un solar de poco más de medio millar de metros cuadrados.

Junto a este sugestivo espacio y las dependencias de la planta noble que le rodean, cabe destacar como elementos más significativos del edificio, la fachada posterior - con el bellísimo umbráculo de madera de caoba-, la planta subterránea y el 'paisaje' de la azotea, donde parecen jugar las veinte chimeneas de obra vista o decoradas, y la esbelta aguja que contiene la linterna que ilumina el gran espacio central, revestida de piedra cocida y coronada por el imaginativo pararrayos-veleta.

Como podremos observar a través del análisis de sus obras que aquí comentamos, la singular arquitectura de Gaudí se fundamenta en la riqueza e imaginación espacial y ornamental pero también en la imaginación constructiva. Imaginación basada en el aprovechamiento hasta el límite de sus posibilidades estéticas $-y$ también estáticas- de los materiales y sistemas constructivos tradicionales o recientes (utilizados de forma tradicional o no) que caen en las manos del arquitecto. (En el Palau Güell, sirvan como muestras de esta imaginación dos hechos analizados por Josep Maria Moreno en su trabajo publicado en este monográfico, la transformación de la cúpula clásica en la linterna cónica y su revestimiento a base de piedra arenisca vitrificada procedente de los hornos de cal amortizados.) El proceso creativo de Gaudí, naturalmente, tampoco puede estar exento de los conflictos derivados de la antinomia básica de la Arquitectura - la tensión entre plástica y construcción - que señalan en su artículo Albert Casals y José Luis González. El trabajo de Carles Buxadé y Joan Margarit que presentamos resumido en forma de artículo, referido a los aspectos globales de la estructura portante del Palau Güell, pone de manifiesto esta tensión.

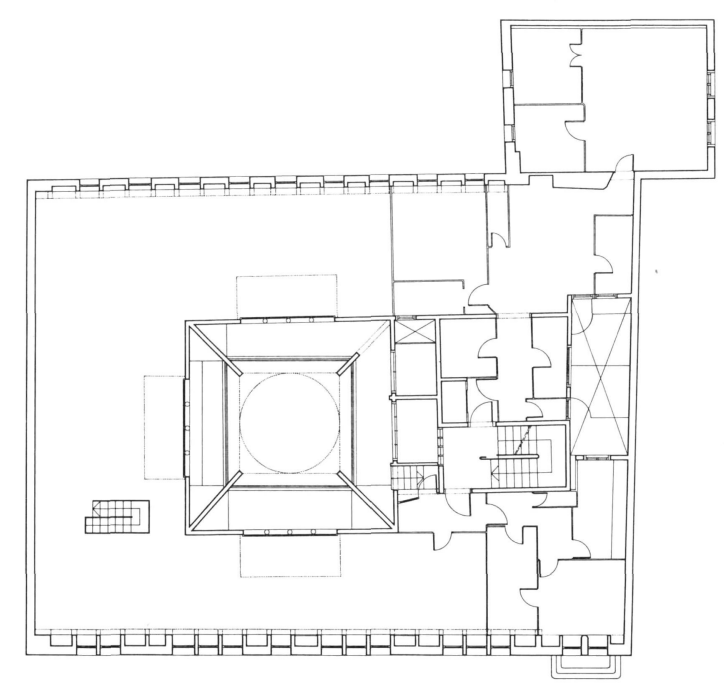

Fig. 8. - Planta desván. Dormitorios de servicio, cocina, lavaderos

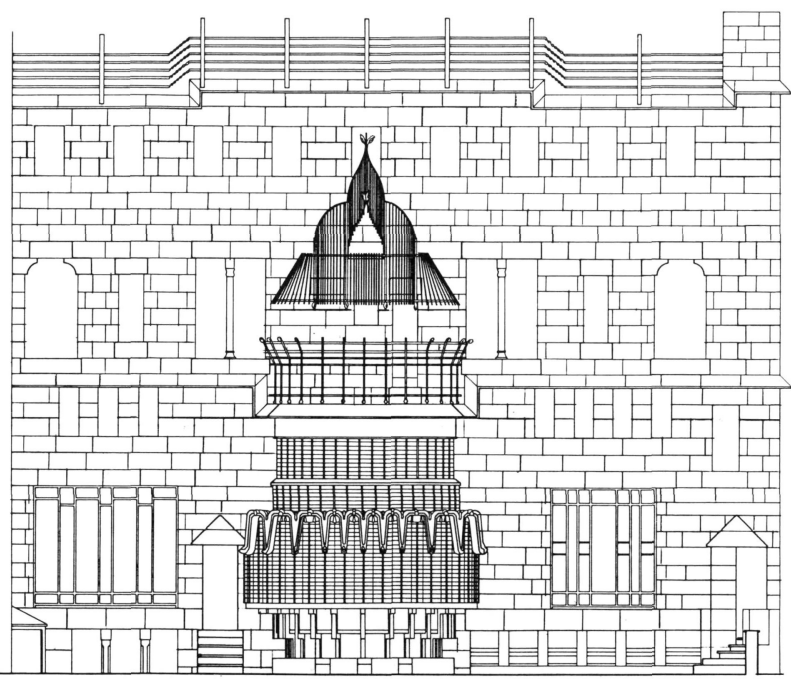

Fig. 9.-Fachada de mediodia (interior del patio de manzana).

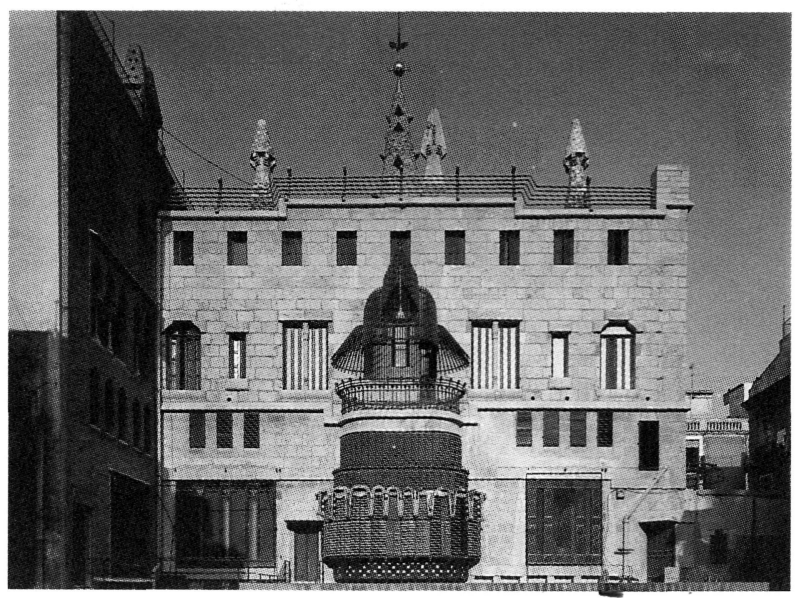

Fig. 10.-Fachada de mediodia, restaurada. (A la izquierda, el cuerpo anexo de servicio). (Foto Jordi Isern, febrero de 1990). 


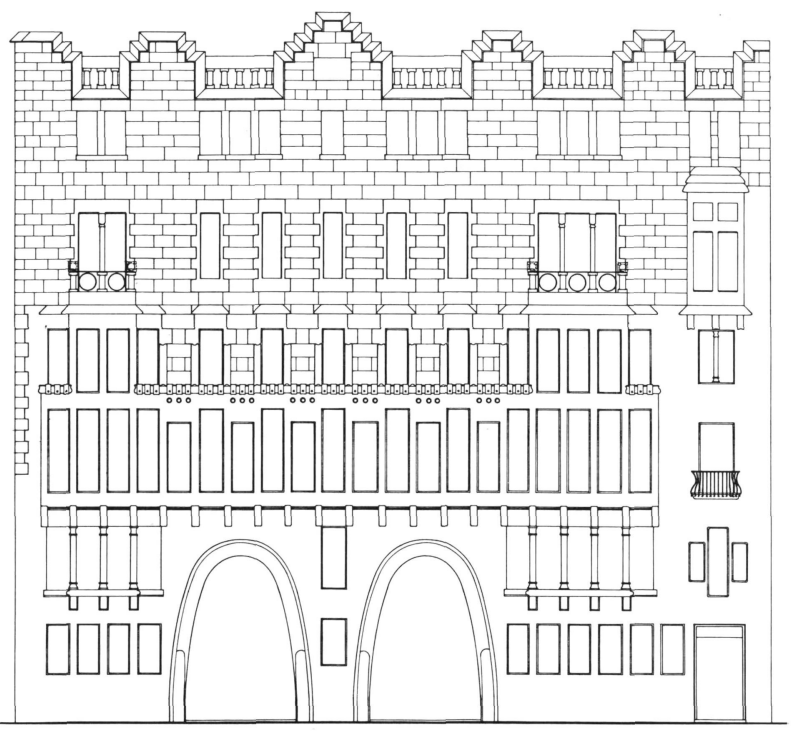

Fig. 11.-Fachada principal.

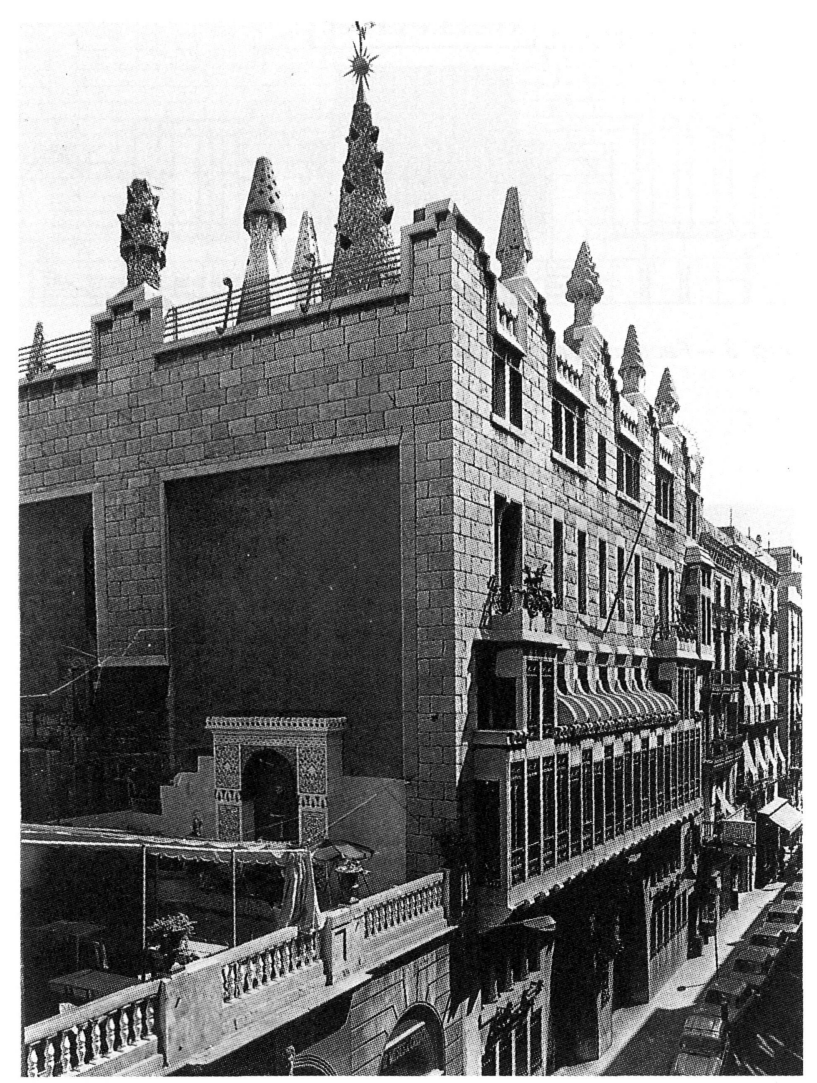

Fig. 12.-Fachada principal y pared medianera. (Foto SPADB, hacia 1970).
Cuando en 1982 el Servicio de Monumentos de la Diputación de Barcelona se hizo cargo de la conservación y restauración del palacio Güell, uno de los primeros trabajos que acometió fue la realización de un dictamen sobre la solidez de la fábrica que fue encargado a los citados catedráticos de la Universidad Politécnica de Cataluña. Este dictamen puso de manifiesto lo que ya intuíamos: Gaudí, en su preocupación por plasmar formal y espacialmente su imaginación desbordada, había supeditado algunos aspectos como los mecanismos de sustentación del edificio. Unos aspectos, no nos engañemos, que siempre han sido subsidiarios.

Conviene insistir en este punto, ya que como podrá comprobar el lector, en el artículo de los profesores Buxadé y Margarit se pone en entredicho la obra de Gaudi a partir de la consideración que merece la estructura de una de sus primeras realizaciones. Se trata, evidentemente, de una valoración hecha desde un aspecto excesivamente particular - un defecto del que les resulta difícil sustraerse a los especialistas - que nos ayuda, eso sí, a clarificar cuáles son las prioridades del joven Gaudí al enfrentarse a su primera obra importante.

Es cierto que el conjunto de elementos sustentantes del Palau Güell es confuso - el excelente análisis de Buxadé y Margarit no dejan lugar a la duda-. Resulta más arriesgado afirmar que el esqueleto de una obra maestra del siglo XIX ha de ser necesariamente una estructura entendida como las entendemos después de las aportaciones de Loos, Mies, o en general del Movimiento Moderno. O dicho de otro modo, es difícil llegar a saber si la concepción del sistema estructural - ahora y siempre - forma parte de los factores esenciales de la arquitectura...

Parece claro que en el palacio de los Güell, la determinación de los elementos que le han de permitir mantener en pie el edificio es para Gaudí un medio más al servicio de lo que resulta realmente fundamental: la construcción de una idea espacial. La valoración de esta obra de juventud hay que hacerla, pues, desde las aportaciones creativas (formales y espaciales) e incluso constructivas, aunque la formulación de una "estructura" global no formara parte de las prioridades de Gaudí en esta obra.

En las páginas que siguen se analizan también los aspectos constructivos de la cubierta del edificio cuya próxima restauración ha inducido a este estudio detallado. Para sucesivos ejercicios la Diputación de Barcelona, en colaboración con el Ministerio de Cultura, ha programado la restauración total del interior que completará la ya mencionada y la de Tás fachadas acometida entre 1983 y 1989. 


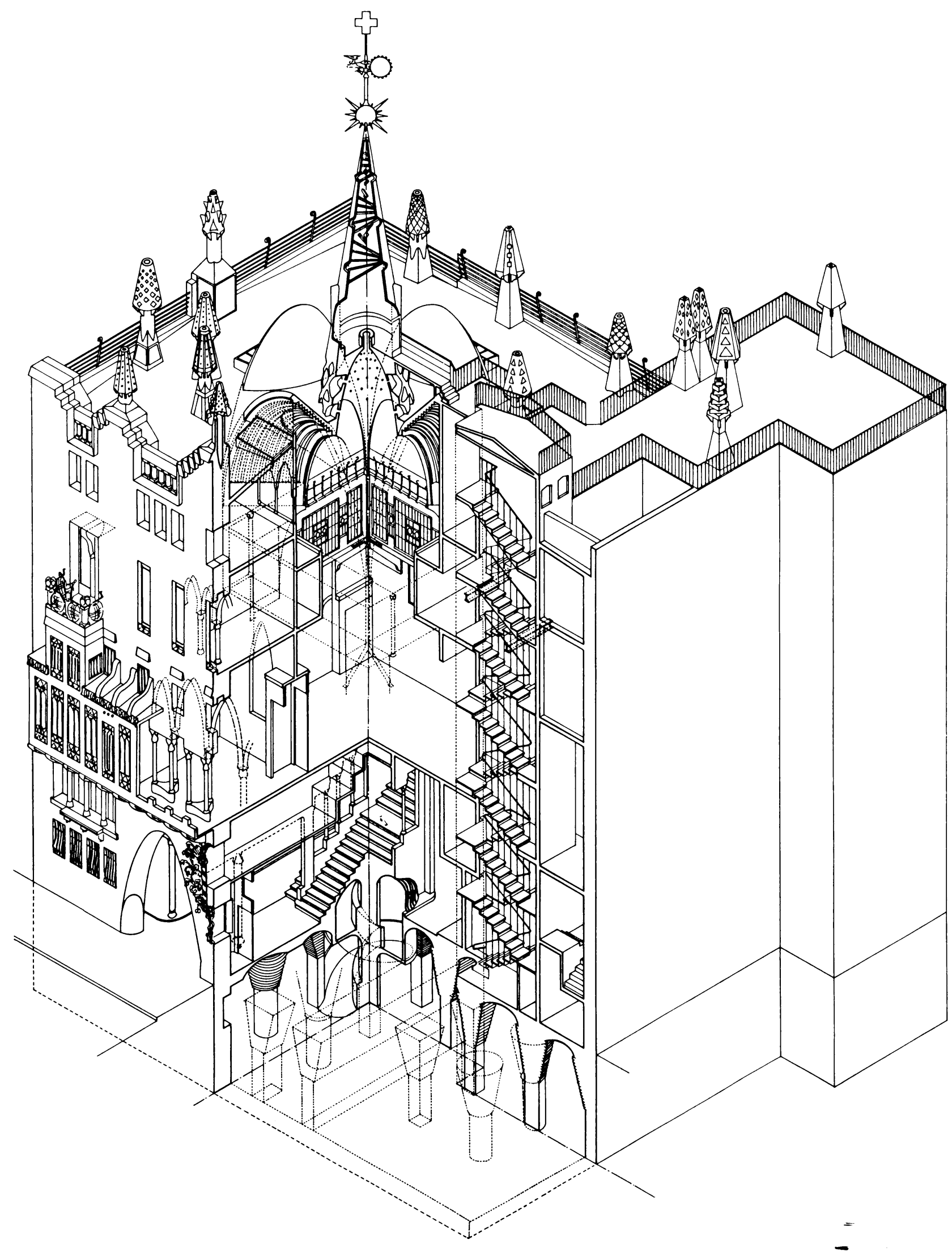

Fig. 13.-Sección axonométrica. Corte por el espacio central, el primer tramo de la escalera principal y la "escalera colgada" o de servicio. (Dibujo Txetxu Sanz). 

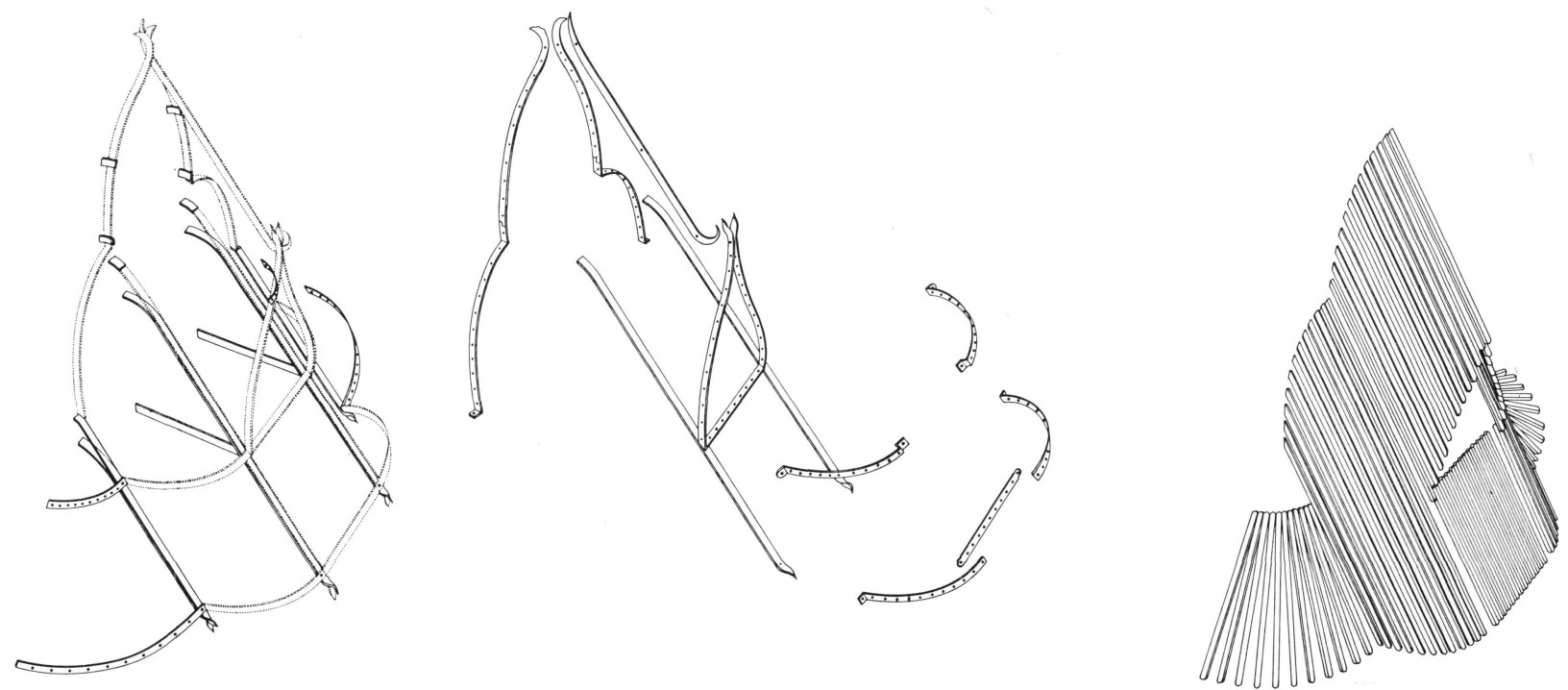

Fig. 14.-Umbráculo de la fachada de mediodia. Disposición de la estructura metálica y los listones de madera de caoba. (Dibujo Txetxu Sanz

Fig. 15.-Umbráculo de la fachada de mediodia, poco después de la restauración. (Foto Jordi Isern, febrero de 1990).

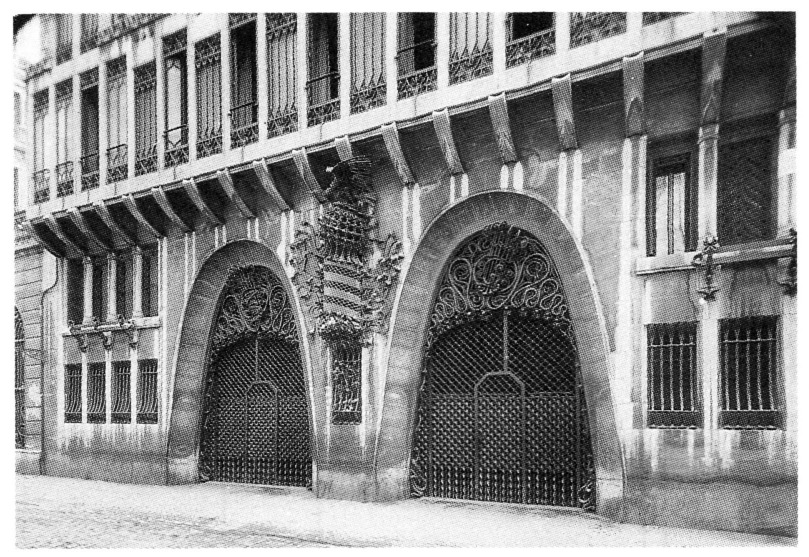

Fig. 16.-Arcos de la fachada

principal. (Foto Arxiu Mas, 1927).

\section{Palau Güell}

Calle Nou de la Rambla, 5 (junto a la Rambla dels Caputxins), Barcelona.

Autor: Antoni Gaudí Cornet, arquitecto (1852-1926)

Fecha de construcción: 1886-1890

Propiedad actual: Diputación de Barcelona

Uso actual: sede provisional del Instituto del Teatro. En obras para ser adaptado como edificio representativo y para mejorar el circuito y las condiciones de visita.

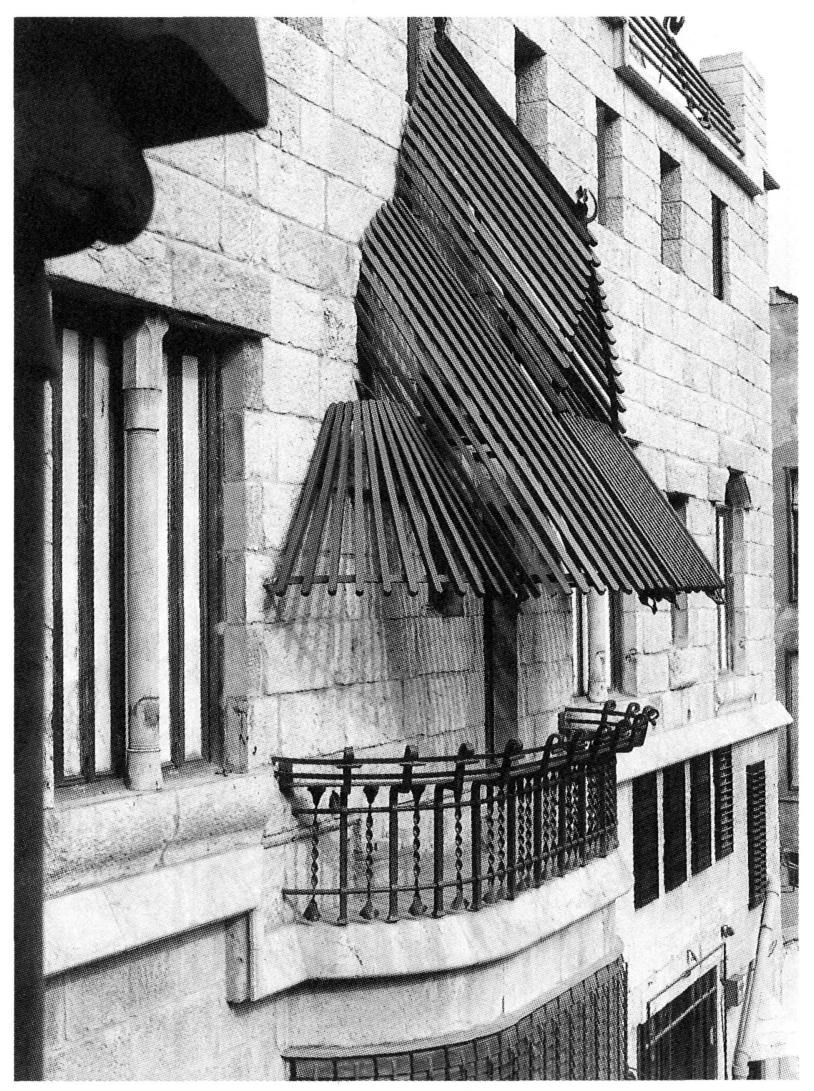

Restauración: Iniciada en 1983. En curso

Promotor: Diputación de Barcelona

Financiación: Diputación de Barcelona, Ministerio de Cultura, Comunidad Europea (CEE)

Técnicos: Antoni González y Pablo Carbó, arquitectos. Josep Maria Moreno y Antoni Rius, aparejadores.

El Palacio Güell es una de las tres obras de Gaudi declaradas Patrimonio de la Humanidad por la UNESCO en 1985. Es monumento nacional desde 1969. 